\title{
$\widehat{A}$ Madridge \\ madridge Journal of Immunology \\ Interconnecting Scientific World
}

Review Article

Open Access

\section{Adolescent Venereal Diseases}

\author{
Siniša Franjićc
}

Faculty of Law, International University of Brcko, Bosnia and Herzegovina, Europe

\section{Article Info}

\author{
*Corresponding author: \\ Siniša Franjić \\ Faculty of Law \\ International University of Brcko \\ Bosnia and Herzegovina \\ Europe \\ Tel: +387-49-49-04-60 \\ E-mail: sinisa.franjic@gmail.com
}

Received: August 1, 2019
Accepted: August 20, 2019
Published: August 27, 2019

Citation: Franjić S. Adolescent Venereal Diseases. Madridge J Immunol. 2019; 3(2): 95-99.

doi: $10.18689 / \mathrm{mjim}-1000122$

Copyright: @ 2019 The Author(s). This work is licensed under a Creative Commons Attribution 4.0 International License, which permits unrestricted use, distribution, and reproduction in any medium, provided the original work is properly cited.

Published by Madridge Publishers

\begin{abstract}
Sexually transmitted diseases are an important issue in the world because to the high incidence and the potential permanent consequences of general and reproductive health. Sexually transmitted diseases are contagious diseases that are transmitted by direct contact during sexual intercourse (vaginal, oral or anal) with an infected person.
\end{abstract}

Keywords: Adolescents; Sexually transmitted diseases; Venereal diseases.

\section{Introduction}

Sexually transmitted diseases present a major public health problem and are among the commonest causes of illness and even death in the world, and have farreaching health, social and economic consequences [1]. Failure to diagnose and treat traditional infections such as gonorrhoea, chlamydia and syphilis can often have a deleterious effect on pregnancy and the newborn, e.g. miscarriage, prematurity, congenital and neonatal infections and blindness. Other complications, particularly in women, such as pelvic inflammatory disease, ectopic pregnancy, infertility and cervical cancer, are large health and social problems. Eighty percent of HIV infection is spread by the sexual route. The recognition that the presence of an STD, particularly genital ulcer disease, can enhance both the acquisition and transmission of HIV by increased shedding of the virus within and from the genital tract, has resulted increasingly in the development of integrated control programmes for HIV and STDs.

Diagnostic possibilities for many sexually transmitted diseases (STDs), particularly gonorrhea and chlamydial infection, have been revolutionized by nucleic acid amplification (NAATs) and hybrid capture $(\mathrm{HC})$ techniques [2]. NAATs and HC have superior sensitivity and specificity compared with culture or other diagnostic tests such as direct fluorescent antibody (DFA) or DNA probe tests. NAATs and HC also allow use of urine and vaginal specimens, in addition to cervical or urethral specimens. Higher initial costs compared with other tests may be offset by reductions in morbidity.

Of the estimated 15 million new cases of sexuallytransmitted infections (STIs) reported annually, adolescent and young adult women (ages 15-24 years) have the highest rates of Chlamydia trachomatis and Neisseria gonorrhoeae infection of any age group [3]. These STIs pose serious long-term consequences for young women because they are precursors of adverse reproductive health outcomes associated with pelvic inflammatory disease (PID), including tubal infertility, ectopic pregnancy, chronic pain, and increased risk of exposure to HIV.

Adolescent and young adult women's exposure to STIs is the result of complex interrelationships among sociodemographic and behavioral risk factors. Sociodemographic risk factors associated with the acquisition of STIs among adolescents and young adults include younger age, female sex, African American race, and urban residence. Behavioral 
risk factors associated with STls are prevalent among adolescents and young adults. These include sexual initiation at age $\leq 15$ years; multiple, sequential, or concurrent sexual relationships; inconsistent use of condoms; characteristics of sex partners, such as older age and minority race or ethnicity; and frequent use of alcohol and illicit substances, which is often associated with sexual risk behaviors.

\section{Adolescents}

Adolescent venereal disease currently is gaining acceptance as a significant topic of concern among public health officials, physicians, and even the public at large [4]. The proper management of the adolescent patient with venereal disease requires consideration of the medical, epidemiologic, and behavioral aspects of venereal disease control.

The young female progresses from the initial appearance of secondary sex characteristics to early sexual maturity. The child's body undergoes complex changes necessary for adult sexual functioning, and the sex drive becomes awakened. Consequences of adolescent sexuality and sexual activity are the increases in teenage pregnancies and teenage sexually transmitted diseases. Premarital intercourse is beginning at younger ages and that the extent of premarital intercourse is probably on the increase.

With this increase in sexual activity by the young, it is not surprising to find that venereal disease statistics also have risen. The increase of Neisseria gonorrhoeae infection in the teenage group has risen much more rapidly than in the general population (one out of every four reported cases are adolescents age 10-19). Syphilis by contrast is less prevalent and only accounts for $0.5 \%$ of adolescent venereal diseases. It is conservatively estimated that in the United States about one adolescent actually becomes infected with either gonorrhea or syphilis each minute. Reasons for this upswing are multiple. First is the changing sexual mores of the teenage population, second, the earlier age of onset of sexual activity, and third, the greater range of sexual partners. A fourth possible reason is the increase in reported case findings, and finally, the superficial knowledge that adolescents have of venereal disease.

Adolescents are particularly at high risk of venereal disease because they are sexually active and are often late in seeking medical attention. They are also less likely than older persons to form a monogomous relationship, and they are also less likely to take appropriate protective measures before engaging in sexual activity. Also, when they become infected, young persons may delay seeking the proper medical attention. This is secondary to their lack of accurate information concerning symptoms, consequences, and the appropriate treatment of sexually transmitted diseases. They also have a mistrust of medical personnel for fear their confidentiality could be violated. Also, the inadequate financial resources they possess often inhibit them from seeking private medical care. Because of the extensive venereal disease epidemic among adolescents, physicians whose practices include this age group should expect to see more venereal diseases.

\section{Sexual Life}

A responsible, safe, and fulfilling sexual life experience requires a positive approach to sexuality and sexual relationships, as well as an understanding ofthe interpersonal, social, and culture factors (e.g., gender violence, discrimination) that may lead to sexual ill-health [5]. Sexual health involves positive sexual expression, coupled with the possibility of satisfying and safe sexual experiences. As adolescents begin to explore their sexuality, including the initiation of sexual activity, they may be at risk for sexual illhealth. Sexually active adolescents may experience a number of short- and long-term consequences. Potential short-term consequences include early pregnancy and/or unsafe abortion, sexually transmitted infections (STIS), and sexual coercion and/or violence. Acquiring an STI during adolescence may lead to long-term consequences. For example, the human papillomavirus (HPV), the most common STI, is associated with the development of cervical, anal, vulvar, vaginal, penile, and oropharyngeal cancers many years after its acquisition and initial diagnosis. Clearly, achieving sexual health among adolescents is a public health priority.

\section{Sexual Activity}

Age and phase of life are key sociodemographic variables that influence contraceptive use [6]. The needs of a sexually active teenager for whom pregnancy might be unwanted or socially stigmatizing are clearly different than those of a woman in her midreproductive years looking to space children, or a woman in later life who has completed all intention of childbearing. In fact, age is directly related to contraceptive utilization, which increases linearly with age. Between ages 40 and $44,75 \%$ of women use contraception, though $8.6 \%$ remain at risk of unintended pregnancy. Many of these women incorrectly believe that they no longer require contraception due to a perceived lack of fertility.

At the younger end of the age spectrum, adolescents have a unique set of barriers that interfere with their engagement in using contraception. Adolescence is defined by psychological maturity that is markedly behind the level of physical maturity. Consequently, adolescents may follow a pattern of cognitive thought that makes them unable to appreciate the long-term consequences of current acts, coupled with a developmental tendency toward risk taking behavior. As a result, they may deny or minimize the risks of pregnancy and fail to properly employ any contraception. In addition, they may lack education about contraceptive options, and not have a family or peer environment that is supportive of contraceptive use. Finally, adolescents may not have access to effective contraception, whether as a result of lack of guaranteed confidentiality and perceived adverse repercussions to asking about access, or as a result of being unable to financially or geographically access contraceptive services at this young age. 
Socioeconomic status is itself a correlate of contraceptive use. Women who come from backgrounds of lower economic class are less likely to use effective contraception, due to a variety of factors including lack of education, distrust of medical providers, poor access to care and provider bias. Improving coverage for contraceptive methods and access to medical care could dramatically affect the reproductive health of poorer populations, and public health studies suggest that women who live in areas where universal coverage is available have lower rates of unintended pregnancy and abortion.

\section{Sexual Partner}

Whether individuals should succumb to their sexual urges outside marriage can be a source of considerable tension, anxiety, and guilt, aggravated by legal and ethical constraints and sexual indulgence between married partners is not free from ethical concerns of mutual respect [7]. Similarly, whether partners have freely consented can be a source of anxiety and self recrimination on ethical and legal grounds. Sexual relations with underage partners, of either sex, can be an obvious legal concern, but modern attention includes relations with elderly voluntary partners affected by degrees of dementia, such as when perhaps Viagra-aided men find same-age companions.

Touching without consent is generally addressed in law relating to assault. Consent to ordinary touching is often implied by conduct, such as when entering a crowded train or sports arena. Sexual touching is more intimate, and sexual assault is usually more heavily punishable than common assault because it affects not only individuals' bodily integrity but also their emotional well-being, dignity, and sense of security. Many legal systems set ages of consent before which adolescents'consent to sexual touching or intercourse is legally invalid, rendering the acts offences. Sexually precocious adolescents may be considered delinquents for consensual relationships, but are increasingly regarded less as offenders than as offended against, by partners and, for instance, by parents' lack of due care. Further, if a sexual partner is less than three years or so older than the other who is underage, this may be seen as misguided sexual curiosity rather than a serious offence. Adolescent girls may be induced to restraint, however, by being made apprehensive of unwed pregnancy if it carries a social stigma.

\section{HPV}

Genital human papillomavirus (HPV) is a viral sexually transmitted disease (STD) of concern because of its high prevalence and causal role in cervical cancer [8]. More than 100 types of HPV have been identified, and types are generally classified as low or high risk, depending on their oncogenic potential. HPV-16 is of particular concern, because it is the most prevalent type of genital HPV and is responsible for $\sim 50 \%$ of cases of cervical cancer worldwide.
Determination of the epidemiology of genital HPV infection has been hindered by suboptimal detection methods. Because HPV is difficult to grow in cell culture, most studies have relied on the detection of HPV DNA on mucosal surfaces (e.g., cervix). However, mucosal DNA does not persist in most persons after infection, and attempts to detect it are prone to sampling error, so there has been interest in approaches-such as serum antibody measurement-that may provide a potentially better assessment of lifetime infection. Results of serologic assays appear to correlate better with cumulative past exposure than do DNA-based approaches; however, although highly type specific, they have variable sensitivity, possibly because of the poor immunogenicity of HPV infections in the superficial epidermis. Seroepidemiologic studies to date have been primarily cross-sectional prevalence studies, have largely focused on women, and have generally found that the seroprevalence of HPV antibody is more strongly associated with lifetime sexual behavior than with more-recent sexual behavior. There are relatively limited data that are relevant to the seroepidemiology of HPV infection in men and few longitudinal studies of HPV antibody that have assessed the persistence and incidence of HPV antibody in men or women.

\section{Chlamydia}

Chlamydia trachomatis is a gram-negative, obligate intracellular bacterium that can infect human ocular, genital tract, and respiratory epithelium. Globally, trachoma (serovars $A, B, C$ ) is the leading cause of infectious blindness [9]. Repeated infections within endemic areas result in chronic inflammation and damaged conjunctiva that leads to trichiasis, scarring, and blindness. Genital infection can cause urethritis and proctitis in men, and urethritis, cervicitis, endometritis, and salpingitis in women with serious sequelae including pelvic inflammatory disease (PID), tubal factor infertility, ectopic pregnancy, and chronic pelvic pain. Furthermore, Chlamydia leads to increased transmission and acquisition of human immunodeficiency virus (HIV). Recent estimates suggest that there are approximately 3.0 million sexually transmitted chlamydial infections in the USA and 90 million new infections globally each year. Genital infection is most prevalent among adolescents and young adults, affecting approximately $4 \%$ of $18-26$-year-olds in the USA. Screening programs can identify and treat at-risk populations; however, these programs are costly and may prevent natural immunity from developing-a concept referred to as the "arrested immunity hypothesis".

Chlamydia trachomatis is the most common cause of bacterial sexually transmitted infection (STI) and is associated with an increased risk of pelvic inflammatory disease, ectopic pregnancy, tubal infertility, and increased susceptibility to human immunodeficiency virus infection [10]. Repeated chlamydial genital infections are common and account for a substantial proportion of incident infections. Repeated infections result from failure of antibiotic therapy or from reinfection due to unprotected sexual contact with either an untreated existing partner or a new infected partner. 
Distinguishing among these possibilities is important to focus treatment recommendations and disease control activities. For example, if many repeated infections are due to antibiotic treatment failure, then better antibiotic treatment regimens are needed. If most are reinfections, then strategies to either expedite partner treatment or screen and treat men in high-risk networks are necessary. The relative frequency of treatment failure and reinfection is not well defined.

\section{Gonorrhea}

Gonorrhea is one of the oldest and best-known STDs [11], caused by a bacterium called Neisseria gonorrhoeae, the disease has been described by medical experts dating as far back as 3500 B.C. The name gonorrhea comes from Latin, and literally means "flowing of the gonads", a reference to the pus that sometimes flows from the genitourinary tract of infected individuals. Over the past 60 years or so in the United States, gonorrhea has come to be known by a wide variety of slang names, such as "the clap", "the drips", "racehorse" and "whites". Despite its potential to cause serious problems, as recently as the 1970s, some American men actually considered getting gonorrhea a sign of manhood.

Neisseria gonorrhoeae, like other members of the Neisseriaceae family of bacteria, normally thrives in the mucous membranes. This particular member is limited to the genitourinary system. Because of its spherical shape, Neisseria gonorrhoeae is sometimes called gonococcus, meaning "the round bacteria of the genitals".

Gonorrhea is almost exclusively spread through sexual contact. In men, the Neisseria gonorrhoeae bacteria enter the urethra when the penis comes in contact with infected vaginal mucus during sexual intercourse, or other fluids during anal intercourse. The bacteria then use special chemicals to bind to the cells of the urethra. In women, the gonorrhea-causing bacteria are generally found lining the cervix, urethra, and vagina. Women typically acquire gonorrhea from sexual contact with the semen of an infected man.

\section{PID}

Pelvic inflammatory disease (PID), an infection of the upper genital tract, is a life-threatening and, unfortunately, common complication of gonorrhea among women [11]. It involves the fallopian tubes, uterus, and ovaries, and occurs in approximately $10 \%$ to $17 \%$ of females who get gonorrhea. The incidence of PID is difficult to track because doctors are not required to report it to local health departments, and most infections these days do not require the patient to be hospitalized. It is estimated that about $20 \%$ of all PID cases occur among adolescent females.

PID begins in the cervix and ascends to the lining of the uterus and to the fallopian tubes, ovaries, and abdomen. The abdominal cavity contains the lower portion of the digestive system, including the intestines, liver, pancreas, spleen, and stomach. A delicate membrane called the peritoneum lines the abdominal cavity and surrounds all of these organs. It is probable that Neisseria gonorrhoeae could leave the fallopian tubes and start growing on the peritoneum. This is followed by inflammation and perforation of the delicate membrane (A perforation is a hole in the membrane that then exposes the internal organs to possible hazards, including pathogenic microbes). After the peritoneum is perforated, all of the organs are vulnerable to destruction by Neisseria gonorrhoeae.

\section{Syphilis}

Syphilis is a transfusion-transmitted infection (TTI) due to a spirocheta called Treponema pallidum [12]. The germ is present in the blood of a contaminated blood donor and infects the recipient. The transmissibility of syphilis by blood transfusion has been frequently reported, chiefly based on animal experiments. Cases of syphilis transmitted by blood have been described in literature, with more than a hundred cases since the first description. The main cases reported were shown to occur when donors were in the primary or secondary stage of the disease. T. pallidum may be found in the blood stream, but levels are variable, and bacteremia is often short-lived even in recent contamination. Moreover, the treponemes are relatively fragile and sensitive to cold; storage below $+20^{\circ} \mathrm{C}$ for more than 72 hours destroys the organism and reduces dramatically the infectious risk. Although clearly potentially infectious, the risk of transmission through the transfusion of blood and blood components stored below $+20^{\circ} \mathrm{C}$ is very low. Platelet concentrates usually stored at a temperature above $+20^{\circ} \mathrm{C}$ or blood directly transfused few hours after collection comprises a higher risk of transmitting syphilis. This is the case in many developing countries with limited blood supply where blood is collected from family donors and frequently transfused in the following hours or days. Thus, the screening test is considered essential as most blood transfusion services are concerned by storage of blood products stored either above $+20^{\circ} \mathrm{C}$ or not stored below $+20^{\circ} \mathrm{C}$ for more than 4 days. Furthermore, it is actually shown that the deferral of blood products screened positive also reduces the risk of contamination from HIV and HBV.

When the germ is transmitted to the recipient, some signs appear a few weeks later notably macular lesions on the palms, headache, arthralgia, fever, headache, peripheral lymphs nodes and more rarely jaundice. For all the reported cases, there was neither a history of venereal disease nor presence of sores on the blood donor at the time of donation. However, many cases were associated with appearance of a sore on the blood donor few days after the donation. Thus, syphilis can be transmitted from donors who are clinically and biologically negative. It is clear that medical selection and mainly information and questionning are essential to identify those who have been exposed to infection during the preceding two months.

Syphilis made sex risky as soon as it was established as a sexually transmitted disease [13]. However, the concerns died down once antibiotics proved successful at fully curing 
the disease. AIDS refueled the idea that sex could have dangerous consequences. However, unlike syphilis, there are no indications of an immediate cure for AIDS, which is elevating societal concerns about the disease. Society is now redefining its attitudes about sex, thanks again to the widespread occurrence of a stigmatic STD. Television shows and movies portraying casual and extramarital sex are gaining much criticism as a result of the spread of AIDS. Such entertainment is being accused of expanding unhealthy attitudes about sex and is being blamed for the high prevalence of unprotected sex. The rise in teen pregnancy and the reoccurrence of STDs are being used as evidence of the consequences of relaxed sexual attitudes. Even safe sex using condoms does not ensure total protection from AIDS. Sex education programs are now being compelled to stress abstinence or alternate forms of sexual pleasure. A further limitation to sexual freedom is the growing number of cases of multiple antibiotic-resistant bacterial STDs. This could raise syphilis to the status of AIDS if it once again proves to be an incurable disease due to antibiotic resistance.

\section{Conclusion}

Sexually transmitted diseases are contagious diseases that are transmitted by direct contact with sex, although this is not the only way to transfer the disease. Causes are infections of viruses, bacteria, fungi and other microorganisms. Most infected are adolescents and young people upto 19 years of age. Sexually transmitted diseases, if they are not recognized in time and are not cured, lead to serious health damage, and some of them are deadly, like AIDS.

\section{References}

1. Adler M. Epidemiology of Sexually Transmitted Diseases and Sexual Health. In: Miller D, Green J (eds). The Psychology of Sexual Health. 2002: 13. doi: $10.1002 / 9780470760109$.ch2

2. Fortenberry JD, Neinstein LS. Overview of Sexually Transmitted Diseases. In: Neinstein LS; Gordon CM, Katzman DK, Rosen DS. Handbook of Adolescent Health Care. Lippincott Williams \& Wilkins, Wolters Kluwer, Philadeplhia, USA. 2009: 521.

3. Boyer CB, Shafer MA, Pollack LM, Canchola J, Moncada J, Schachter J. Sociodemographic Markers and Behavioral Correlates of Sexually Transmitted Infections in a Nonclinical Sample of Adolescent and Young Adult Women. J Infect Dis. 2006; 194(3): 307-315. doi: 10.1086/506328

4. Bolton GC. Venereal Disease in Adolescents. In: Bongiovanni AM (ed). Adolescent Gynecology. 1983: 145-146. doi: 10.1007/978-1-4615-8324-0_7

5. Scott-Sheldon LAJ, Johnson BT. The Sexual Health of Adolescents When, Where, and Why Adolescents Use Contraceptives. In: Bromberg DS, O'Donohue WT (eds). Handbook of Child and Adolescent Sexuality Developmental and Forensic Psychology, Academic Press, Elsevier, Oxford, UK. 2013: 221-222.

6. Schaffir J. Psychosocial Aspects of Fertility Control. In: Edozien LC, O'Brien PMS (eds). Biopsychosocial Factors in Obstetrics and Gynaecology. Cambridge University Press, Cambridge, UK. 2017: 199-200.

7. Dickens BM, Cook RJ. Legal and Ethical Factors in Sexual and Reproductive Health. In: Edozien LC, O'Brien PMS (eds). Biopsychosocial Factors in Obstetrics and Gynaecology, Cambridge University Press, Cambridge, UK. 2017: 209.
8. Thompson $\mathrm{DL}$, Douglas JM Jr, Foster $\mathrm{M}$, et al. Seroepidemiology of Infection with Human Papillomavirus 16, in Men and Women Attending Sexually Transmitted Disease Clinics in the United States. J Infect Dis. 2004; 190(9): 1563-1574. doi: 10.1086/423817

9. Poston TB, Darville T. Chlamydia trachomatis: Protective Adaptive Responses and Prospects for a Vaccine. In: Häcker G (ed). Biology of Chlamydia. Springer International Publishing AG, Cham, Switzerland 2018: 217.

10. Batteiger $\mathrm{BE}, \mathrm{Tu} \mathrm{W}$, Ofner $\mathrm{S}$, et al. Repeated Chlamydia trachomatis Genital Infections in Adolescent Women. J Infect Dis. 2010; 201(1): 42-51. doi: $10.1086 / 648734$

11. Kollar L, Shmaefsky BR. Gonorrhea. Chelsea House Publishers, Philadelphia, USA. 2005; 9: 73.

12. Tagny CT. Syphilis and Blood Safety in Developing Countries. In: Sato NS (ed). Syphilis - Recognition, Description and Diagnosis. InTech, Rijeka, Croatia. 2011: 123-124. doi: 10.5772/21499

13. Shmaefsky B. Syphilis. Chelsea House Publishers, New York, USA. 2003: 111. 